\title{
Endometrial Mesenchymal Stem Cells Isolated from Menstrual Blood by Adherence
}

\author{
Xue Du, ${ }^{1}$ Qing Yuan, ${ }^{1}$ Ye Qu, ${ }^{2}$ Yuan Zhou, ${ }^{3}$ and Jia Bei ${ }^{4}$ \\ ${ }^{1}$ Department of Obstetrics \& Gynecology, General Hospital, Tianjin Medical University, Tianjin 300052, China \\ ${ }^{2}$ Department of Pathogenic Biology and Immunology, Logistics College of Chinese People's Armed Police Forces, Tianjin 300309, China \\ ${ }^{3}$ State Key Laboratory of Experimental Hematology, Institute of Hematology and Blood Diseases Hospital, \\ Chinese Academy of Medical Sciences and Peking Union Medical College, Tianjin 300020, China \\ ${ }^{4}$ Department of Biomedical Engineering, University of Dundee, Dundee DD1 4HN, UK
}

Correspondence should be addressed to Xue Du; lanlandetommao@163.com and Yuan Zhou; yuanzhou@ihcams.ac.cn

Received 23 March 2015; Accepted 26 August 2015

Academic Editor: Thomas Ichim

Copyright (c) 2016 Xue Du et al. This is an open access article distributed under the Creative Commons Attribution License, which permits unrestricted use, distribution, and reproduction in any medium, provided the original work is properly cited.

Objective. To find a convenient and efficient way to isolate MSCs from human menstrual blood and to investigate their biological characteristics, proliferative capacity, and secretion levels. Methods. MSCs were isolated from menstrual blood of 3 healthy women using adherence. Cell immunological phenotype was examined by flow cytometry; the adipogenic, osteogenic, and chondrogenic differentiation of MSCs was examined by Oil-Red-O staining, ALP staining, and Alcian Blue staining, respectively; and the secretion of cytokines, including vascular endothelial growth factor (VEGF), hepatocyte growth factor (HGF), and insulin-like growth factor1 (IGF-1), was detected using enzyme-linked immunosorbent assay. Results. MB-MSCs were successfully isolated from human menstrual blood using adherence. They were positive for CD73, CD105, CD29, and CD44, but negative for CD31 and CD45. The differentiated MB-MSCs were positive for ALP staining, Oil-Red-O staining, and Alcian Blue staining. In addition, they could secrete antiapoptotic cytokines, such as VEGF, IGF-1, and HGF. Conclusion. It is feasible to isolate MSCs from human menstrual blood, thus avoiding invasive procedures and ethical controversies. Adherence could be a promising alternative to the density gradient centrifugation for the isolation of MSCs from menstrual blood.

\section{Introduction}

Stem cells have been a topic of considerable interest in the biomedical field over recent years. Stem cells are undifferentiated cells that have a high proliferative potential, substantial self-renewal capacity, and the potential to differentiate into various specialized cell types. Therefore, stem cell therapy has emerged as a promising therapeutic strategy for traumas, immunodeficiency, and hereditary diseases due to their differentiation capacity and plasticity.

Stem cells can be broadly divided into embryonic and adult types. Embryonic stem cells (ESCs) are pluripotent stem cells derived from the inner cell mass of a blastocyst, an early-stage embryo. ESCs exhibit remarkable long-term proliferative potential and capacity for self-renewal, and they are also distinguished by their ability to differentiate into any cell type both in vitro and in vivo. Human ESCs were first derived from mouse embryos in 1981. However, major obstacles remain with the isolation and clinical use of ESCs due to teratoma formation and ethical controversies over the creation, usage, and destruction of human embryos.

Adult stem cells (ASCs) are undifferentiated cells found among differentiated cells of a specific tissue, including bone marrow, peripheral blood, umbilical cord blood, fat, brain, thymus, muscle, liver, and lung. It is well known that ASCs are typically in a dormant state under normal circumstances and start to proliferate and differentiate in response to pathological disorders and external stimuli. ASCs have a controlled behavior and are multipotent as compared to pluripotent capacity of ESCs, thus making them an attractive option for cell-based therapy. ASC treatments have been successfully used to treat Parkinson's disease [1], repair the kidney, and 
improve function in acute renal failure [2,3] and liver injuries of different etiologies [4]. However, the use of ASCs can be limited by their availability, invasiveness of extraction, and in some cases limited proliferative capacity. Although mesenchymal stem cells (MSCs) from bone marrow have been regarded as good candidates for cell therapy, the sampling method is invasive through bone marrow aspiration. Despite their therapeutic potential, MSCs are very rare and a typical adult bone marrow aspirate yields very few MSCs (roughly 1 out of every 10,000 cells) [5].

Human menstrual blood might be an excellent source of ESCs. Chan et al. provided the first evidence for the existence of epithelial and stromal stem cells in human endometrium [6]. In 2004, Chan et al. [6] reported for the first time the isolation of stem cells directly from human endometrium. In 2007, Meng et al. [7] isolated MSCs from menstrual blood. Menstrual blood is easily accessible, renewable, and inexpensive. MSCs isolated from menstrual blood (MBMSCs) may offer certain advantages, including noninvasive procedures, in vitro culture, and ethically nonproblematic availability. However, research on this new source of MBMSCs is still in its infancy, and more research needs to be carried out on the isolation, secretion, and identification of MB-MSCs. In this study, MSCs were isolated from menstrual blood based on their strong adherence to plastic or glass, and their biological characteristics, proliferative capacity, and secretion levels were also investigated. The results of this study will help us to better understand the isolation of MSCs from human menstrual blood.

\section{Materials and Methods}

2.1. Collection of Menstrual Blood. Cell donation was approved by Local Ethical Committee for research purposes, and written informed consent was obtained from each donor. Infectious pathology was excluded by the HIV, HCV, and HBV tests. Menstrual blood was collected from three healthy female volunteers aged 25-35 years during the first 3 days of the cycle using a menstrual cup (Green Donna, Guangzhou Meifanle Rubber Products Co., Ltd., China). The menstrual cup was retained in the vagina for a mean of 3 hours (2-4 hours). The menstrual blood was transferred into phosphate buffered saline (PBS) containing $1 \%$ penicillin and streptomycin, maintained at $4^{\circ} \mathrm{C}$, and transported to the laboratory within $24 \mathrm{~h}$ of collection.

2.2. Isolation and Culture of Human MB-MSCs. The menstrual blood was transferred to a $15 \mathrm{~mL}$ centrifuge, and red cells were depleted by Red Blood Cell Lysis Buffer (SigmaAldrich, St. Louis, MO, USA). Cells were counted by blood counting chamber. Cells were seeded at $5 \times 10^{5}$ cells/well in 6-well plates (Corning, New York, USA) and cultured in DMEM-F12 supplemented with $10 \%$ FBS, streptomycin $(100 \mathrm{U} / \mathrm{mL})$ and penicillin $(100 \mathrm{U} / \mathrm{mL})$ (Gibco, Grand Island, $\mathrm{NY})$ at $37^{\circ} \mathrm{C}$ in humidified air of $5 \% \mathrm{CO}_{2}$. Culture medium was refreshed every 3-4 days. When the cells reached $80 \%$ confluence, they were passaged using $0.25 \%$ Trypsin (Gibco, Grand Island, NY, USA). All experiments were performed with cells at passage 3 from the 3 donors.
2.3. Immunophenotypic Analyses of MB-MSCS. Cells were harvested in $0.25 \%$ Trypsin and fixed in ice-cold $2 \%$ formaldehyde for $30 \mathrm{~min}$. Then, the fixed cells were washed in PBS, adjusted to a density of $1 \times 10^{9} / \mathrm{L}$, and incubated with $1 \mu \mathrm{L}$ of antibodies (CD45 FITC, CD31 PE, CD44 APC-CY7, CD29 APC, CD105 Percp-Cy5.5, and CD73 PE) (BD Biosciences, San Jose, USA) at $4^{\circ} \mathrm{C}$ for $30 \mathrm{~min}$. After that, cells were washed with PBS and centrifuged at $1500 \mathrm{r} / \mathrm{min}$ for $5 \mathrm{~min}$. The supernatant was discarded, and the cells were resuspended with PBS. The antibody-labeled cells were analyzed using FACSCalibur flow cytometry (BD Biosciences, San Jose, CA), and the results were processed using Cell Quest.

\subsection{Multilineage Differentiation of MB-MSCS. MB-MSCs} were differentiated into adipogenic, chondrogenic, and osteogenic lineages to assess their differentiation capacities in vitro.

2.4.1. Adipogenic Differentiation. MB-MSCs were seeded at $2 \times 10^{5}$ cells/well in a 6 -well chamber slide with $3 \mathrm{~mL}$ media per well. Upon reaching $90 \%$ confluence, the medium was replaced with adipogenic medium containing DMEMF12, $10 \%$ FBS, $10^{-6} \mathrm{~mol} / \mathrm{L}$ dexamethasone, $0.5 \mathrm{mmol} / \mathrm{L}$ isobutyl methylxanthine, $10 \mu \mathrm{g} / \mathrm{mL}$ insulin, and $60 \mu \mathrm{mol} / \mathrm{L}$ indomethacin (Sigma-Aldrich, St. Louis, MO, USA) and cultured for 14 days with medium changes every 3-4 days.

The adipogenic capacity of MB-MSCs was evaluated by Oil-Red-O staining. Cells were fixed with $4 \%$ paraformaldehyde for $30 \mathrm{~min}$, washed with water three times, and then incubated for $30 \mathrm{~min}$ with a filtered $0.5 \%$ Oil-Red (SigmaAldrich Corp., St. Louis, MO, USA) solution in 60\% isopropanol. Cells were washed with water several times prior to photographing. Oil-Red O-positive cells were determined by cytoplasmic fat globule cells.

2.4.2. Osteogenic Differentiation. MB-MSCs were seeded at $4 \times 10^{5}$ cells/well in a 6 -well chamber slide with $3 \mathrm{~mL}$ media per well. After cells adhered overnight, the medium was replaced with osteogenic medium containing DMEM-F12, $10 \% \mathrm{FBS}, 10^{-8} \mathrm{~mol} / \mathrm{L}$ dexamethasone, $10 \mathrm{mmol} / \mathrm{L} \beta$-glycerol phosphoric acid, and $100 \mathrm{mmol} / \mathrm{L}$ ascorbic acid (SigmaAldrich St. Louis, MO, USA), and cultures were continued for another 7 days.

The osteogenic capacity of MB-MSCs was evaluated by ALP staining. Cells were fixed in $4 \%$ paraformaldehyde for $30 \mathrm{~s}$ at room temperature, washed with water three times, and then incubated for $30 \mathrm{~min}$ with staining solution, which was prepared by dissolving Fast Blue RR Salt (Sigma-Aldrich, St. Louis, MO, USA) in $48 \mathrm{~mL}$ of $\mathrm{dd}_{2} \mathrm{O}$ and $2 \mathrm{~mL}$ of Naphthol AS-MX Phosphate Alkaline (Sigma-Aldrich, St. Louis, MO, USA). Then, cells were washed with PBS and incubated for 30 min with Mayer's Hematoxylin (Sigma-Aldrich, St. Louis, MO, USA). The stained cells were washed and imaged under microscope. Purple staining indicated the synthesis of ALP by osteoblasts.

2.4.3. Chondrogenic Differentiation. MB-MSCs were seeded at $4 \times 10^{5}$ cells $/ \mathrm{mL}$ in a 6 -well chamber slide with $3 \mathrm{~mL}$ media per well. After cells adhered overnight, the medium was 
replaced with achondrogenic medium containing DMEMF12, $10 \%$ FBS, $10^{-8} \mathrm{~mol} / \mathrm{L}$ dexamethasone, $10 \mathrm{mmol} / \mathrm{L} \beta$ glycerol phosphoric acid, $100 \mathrm{mmol} / \mathrm{L}$ ascorbic acid, and $10 \mathrm{ng} / \mathrm{mL}$ TGF $\beta-3$ (Sigma-Aldrich, St. Louis, MO, USA). Cultures were allowed to develop for 21 days with medium changes every 3-4 days and were stained by Alcian Blue. Briefly, cells were washed with PBS and fixed with $4 \%$ paraformaldehyde for $30 \mathrm{~min}$. After fixation, cells were washed with PBS and stained with 1\% Alcian Blue solution (Sigma-Aldrich Corp., St. Louis, MO) in the dark overnight at room temperature. Then, cells were washed three times with PBS and visualized under a light microscope. Blue staining indicated synthesis of proteoglycans by chondrocytes.

2.5. Cell Growth Curve Analysis. Cells at P3, P10, and P15 were seeded at $1.0 \times 10^{3}$ cells/well in 96-well plates (Corning, New York, USA) and cultured in DMEM-F12 supplemented with $10 \%$ FBS, $1 \%$ streptomycin, and $1 \%$ penicillin. After $1,3,5,7$, and 9 days, cell proliferation was analyzed by a CCK-8 kit. Briefly, 10\% CCK-8 solution (Beyotime, Shanghai, China) was added to each culture and incubated for $4 \mathrm{~h}$. Then, optical densities (OD) were measured at $450 \mathrm{~nm}$ using an ELISA plate reader (BioTek, Highland Park, USA).

2.6. Enzyme-Linked Immunosorbent Assay (ELISA). MBMSCs were seeded at $5.0 \times 10^{5}$ cells/well in 6 -well plates. Complete medium replacement was performed when cells reached $90 \%$ confluence. Within $48 \mathrm{~h}$, the cells were spun down in a centrifuge, and the supernatant was collected to measure the secretion of growth factors, including vascular endothelial growth factor (VEGF), hepatocyte growth factor (HGF), and insulin-like growth factor-1 (IGF-1) according to manufacturer's protocols (Ruikang Bo, Tianjin, China). Optical densities (OD) were measured at $450 \mathrm{~nm}$ using an ELISA plate reader.

2.7. Statistical Analysis. All analyses were performed using SPSS statistical software (version 13.0; SPSS Inc., Chicago, IL). Results are expressed as mean \pm standard deviation. Significance of difference between groups was assessed by $t$-test or ANOVA, and regression analysis was performed. A one-tail $P$ value of $<0.05$ was considered as statistically significant.

\section{Results}

3.1. Isolation and Culture of MB-MSCs. MB-MSCs adhered on day 6 after two changes of medium, and some nonadherent hematopoietic cells were present in the culture (Figure 1(a)). After initial plating was removed with sequential exchange of culture medium, many colony forming unit-fibroblast (CFUF) colonies were formed after $8 \mathrm{~d}$ of incubation (Figure 1(b)). Cell morphology varied from spindle-shaped to triangular arranged in an unorganized pattern. The number of colonies increased gradually to $80 \%$ confluence after $15 \mathrm{~d}$ of seeding. However, after 2-3 subcultures, the cells reached $90 \%$ confluence only for $5 \mathrm{~d}$ (Figure 1(c)).
3.2. Cell Surface Antigen Expression. The immunophenotypic analysis (Figure 2) showed that P3 MB-MSCs were positive for CD73 (87.4\%), CD105 (96.2\%), CD29 (99.4\%), and CD44 (99.8\%), but negative for CD45 (0.497\%) and CD31 (0.236\%).

3.3. Multilineage Differentiation of MB-MSCSs. MB-MSCs cultured in medium supplemented with differentiation factors could differentiate into mesenchymal lineages, such as osteoblasts, adipocytes, and chondrocytes. The adipogenic differentiation was demonstrated by Oil-Red-O staining on day 14 (Figure 3(a)), and adipocytes were easily identified morphologically by the presence of lipid vacuoles in the cytoplasm. During osteogenic stimulation, MB-MSCs continued to proliferate and changed in morphology. On day 14, almost all alkaline phosphatases in the cytoplasm were stained to be purple (Figure 3(b)). The chondrogenic potential of MBMSCs was characterized by Alcian Blue staining after 21 days of culture, which revealed a deposition of proteoglycans (Figures 3(c) and 3(d)).

3.4. Cell Proliferation Assay. The growth of MB-MSCs was analyzed at early (P3 and P10) and late (P15) passages, and cell proliferation was monitored for 9 days after seeding (Figure 4). The growth of P3, P10, and P15 MB-MSCs all showed a logistic (S-shaped) growth pattern that increased very slowly during the first 1-2 days, followed by an exponential rise from day 2 till day 6 and a stationary phase thereafter. No significant difference in absorbance was observed between P3 and P10 MB-MSCs, but the absorbance of P15 passage was significantly lower. Thus, it could be concluded that there was no difference in cell proliferation between P3 and P10 MB-MSCs, indicating that MB-MSCs still had a strong proliferative potential at P10.

3.5. Secretion of Growth Factors. The ELISA analysis showed that the secretion of growth factors was undetectable in the control group (data not shown), but a large amount of angiogenic and antiapoptotic factors was secreted from MB-MSCs after 3 days of culture, which were $122.953 \pm$ $4.377 \mathrm{pg} / \mathrm{mL}$ for VEGF, $4926.938 \pm 890.475 \mathrm{pg} / \mathrm{mL}$ for HGF, and $1.77 \pm 0.027 \mathrm{ng} / \mathrm{mL}$ for IGF-1, respectively.

\section{Discussion}

Human endometrium is composed of functional and basal layer. The endometrium is about $0.5-1 \mathrm{~mm}$ following menstruation, whereas it is about $10-12 \mathrm{~mm}$ at the end of the cycle. Thus, it is hypothesized that endometrial stem/progenitor cells may play a key role in mediating endometrial repair and subsequent tissue regeneration following menstruation [8]. Chan et al. provided the first evidence for the existence of ESCs in endometrium [6]. However, a potential problem with acquisition of human endometrium is that it is a very invasive procedure. In the recent years, a number of studies have shown that menstrual blood contains a unique population of cells with properties similar to ASCs $[7,9,10]$. An advantage of MB-MSCs is their easy availability, thus avoiding invasive procedures and ethical controversies. In addition, MB-MSCs 


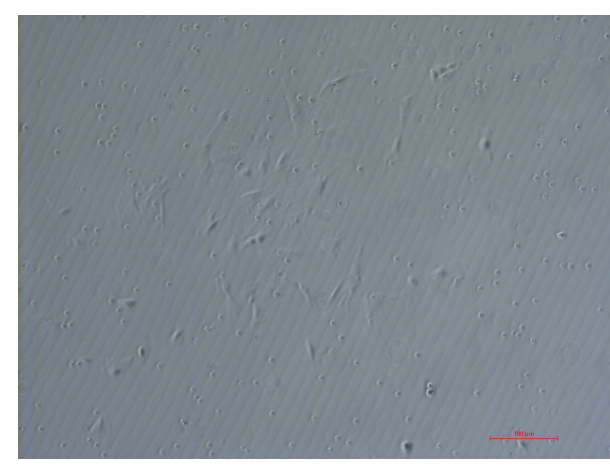

(a)

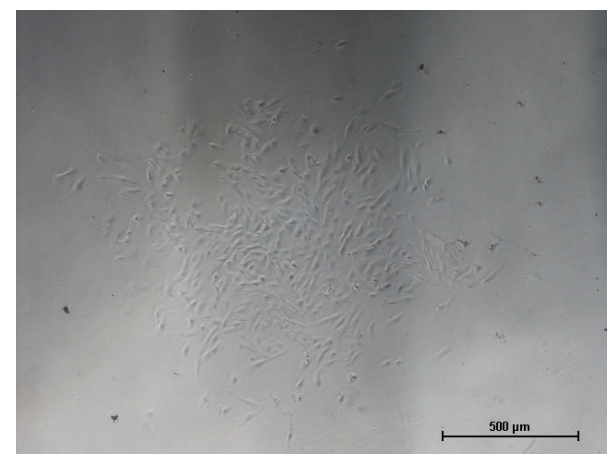

(b)

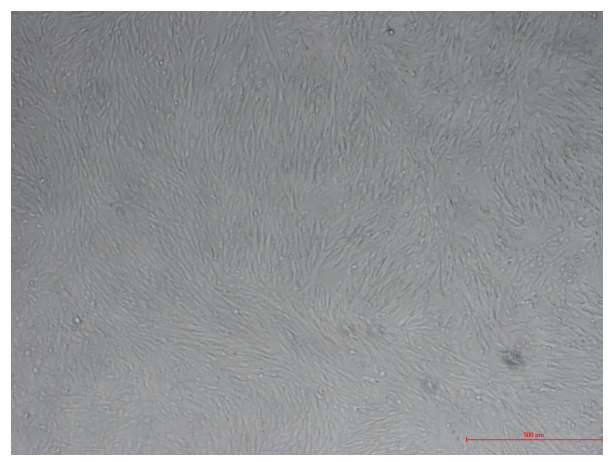

(c)

Figure 1: The morphology of cultured MB-MSCs. (a) The morphology of cultured MB-MSCs at 6 day. (b) Primary cell culture showing initial colony forming unit-fibroblast (CFU-F) on day 8. (c) Cells reached $85-90 \%$ confluence at passage 3.

hold great promise to avoid teratoma formation and immune response of ESCs, and they are also highly expandable in vitro and possess pluripotency. For these reasons, MB-MSCs are increasingly used in a variety of clinical applications, such as stroke [11] and myocardial infarction [12].

MSCs can be isolated from different sources. MSCs may be derived from residual fetal stem cells [13], and there are several lines of evidence indicating that bone marrow-derived stem cells may also populate the endometrium $[14,15]$. It is postulated that most MSCs are located in blood before birth, and some are found in perivascular locations with a unique potential to differentiate into mesenchymal tissue. Later MSCs can migrate to liver, spleen, and bone marrow. After birth, MSCs mainly reside in bone marrow but may also be present in menstrual blood, cord blood, fat, brain, thymus, muscle, liver, and lung [16-19]. Bone marrow stem cells home in on sites of tissue damage and incorporate into various organs, contributing to angiogenesis and/or transdifferentiating into the cells of the new tissue in which they reside [20].

In this study, we have shown that MSCs could be isolated from menstrual blood based on their strong adherence properties. Nonadherent endothelial cells and fibroblasts were removed with sequential change of culture medium. By day 6 , cells adhered, and cell morphology varied from spindleshaped to triangular. The spindle-shaped cells accounted for the majority of cells and had CFU activity by forming large and small CFU-F. It was also observed that cells expressed high levels of CD73, CD90, CD29, CD73, and CD90, the main stem cell markers [21]. But they lacked CD45, a hematopoietic stem cell marker, and CD31, a vascular endothelial cell marker, which was also in agreement with previous studies $[7,9]$.

As no ideal marker of MB-MSCs has been discovered, there is a need for further identification of multilineage differentiation. MSCs cultured in vitro in adipogenic, osteogenic, and chondrogenic medium differentiate into adipocytes, osteocytes, and chondrocytes, respectively. Adipogenic differentiation was evidenced by the presence of lipid vacuoles in the cytoplasm by Oil-Red-O staining. Osteogenic differentiation was evidenced by ALP staining. Differentiation of chondrocytes from MB-MSCs was examined cytochemically with respect to the presence of proteoglycan (Alcian Blue positive). In this study, we found that not all MB-MSCs could differentiate, probably due to the stimulation of chemical reagents, or that MSCs were in different stages of differentiation. It has been demonstrated that most MB-MSCs had the ability to differentiate into cardiac muscle cells and myocytes $[22,23]$. Finally, we showed that MB-MSCs had multilineage differentiation potential.

In this study, MSCs were isolated from menstrual blood using adherence of whole menstrual blood, which is capable of maintaining the stemness and differentiation ability of MSCs. In addition, it is more convenient and efficient than Ficoll density gradient centrifugation. Density gradient centrifugation is a common method used for the separation 

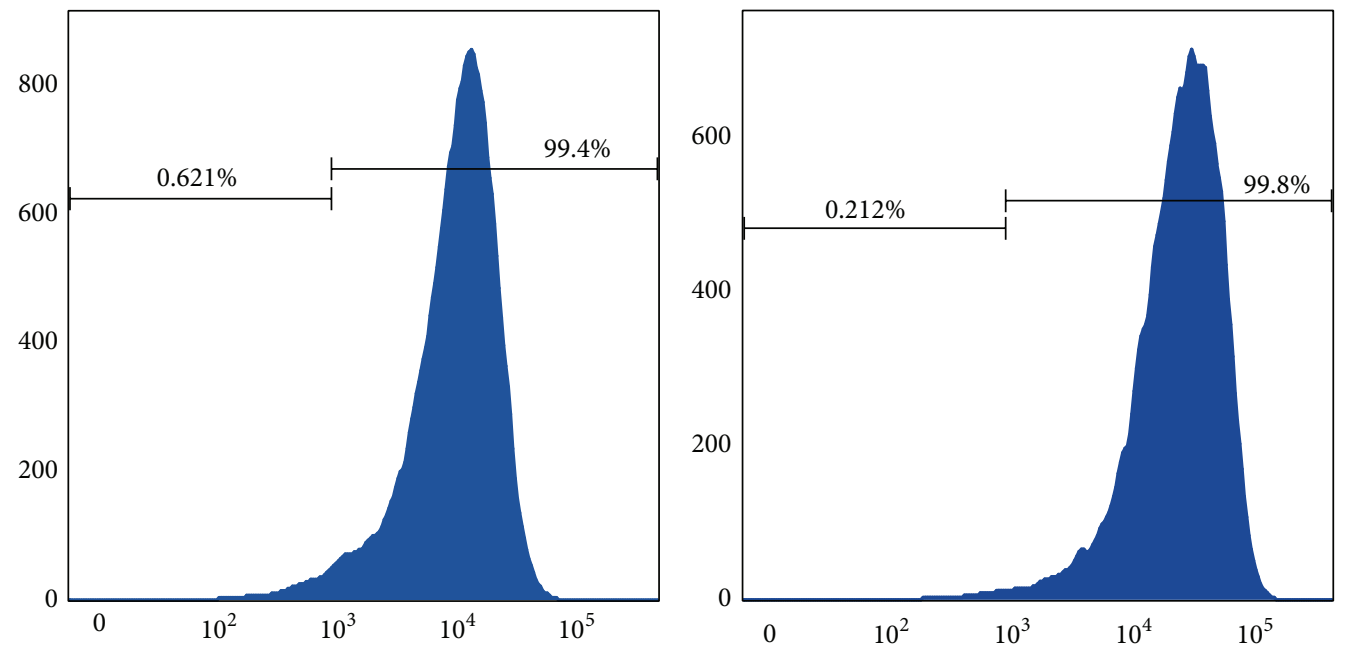

CD29
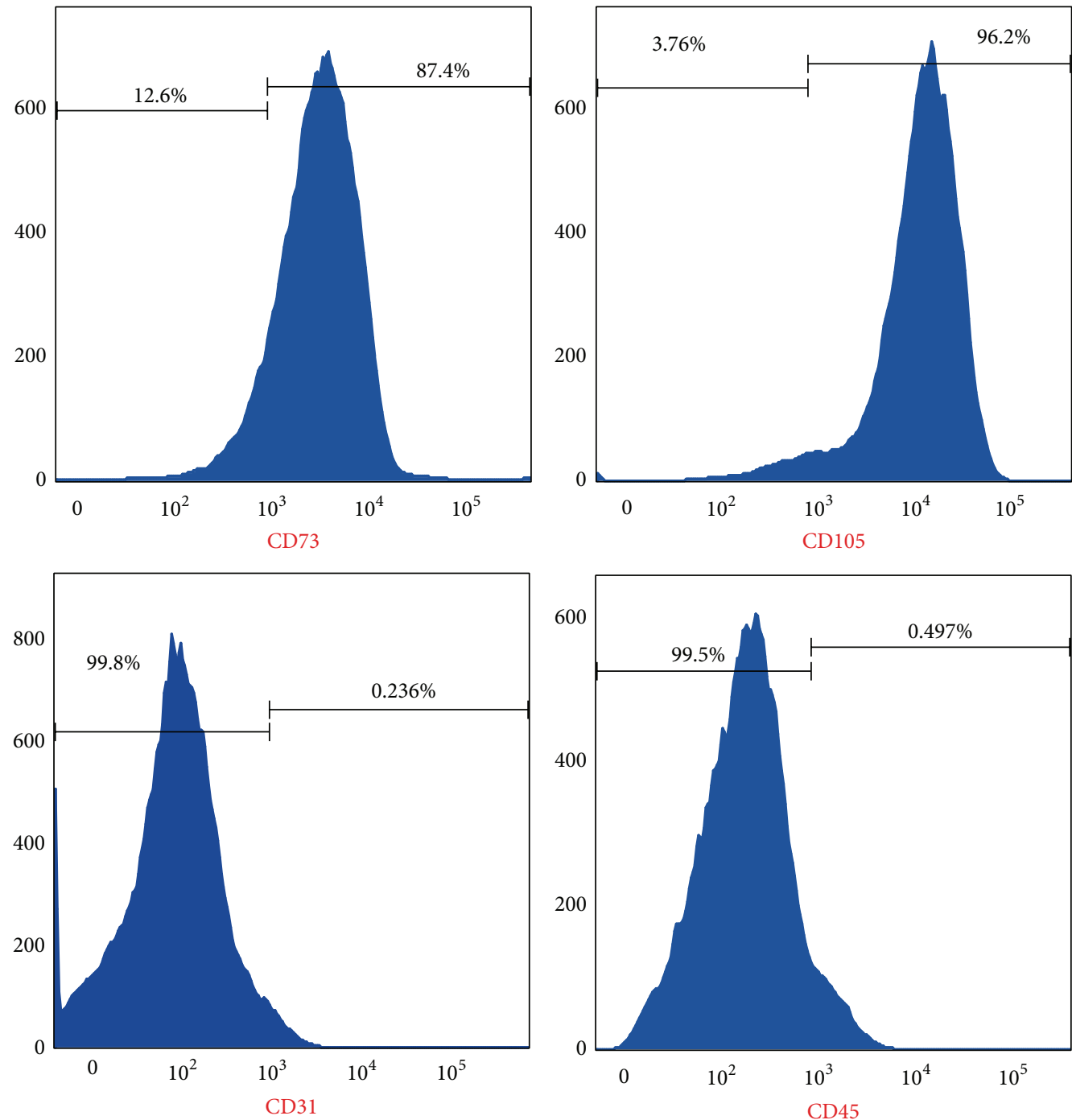

FIGURE 2: Expression of surface markers of P3 MB-MSCs. Percentages are referring to the average positivity for each marker. 


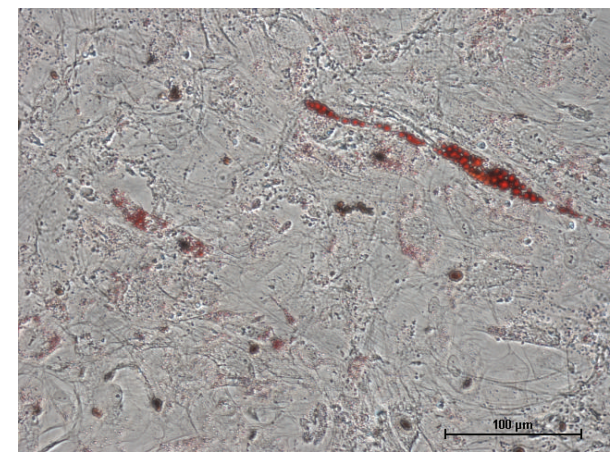

(a)

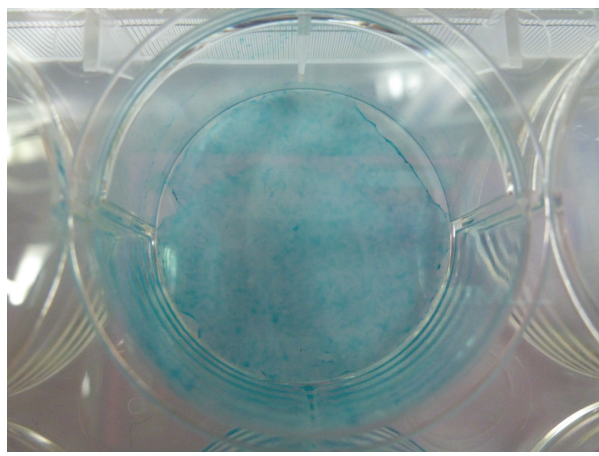

(c)

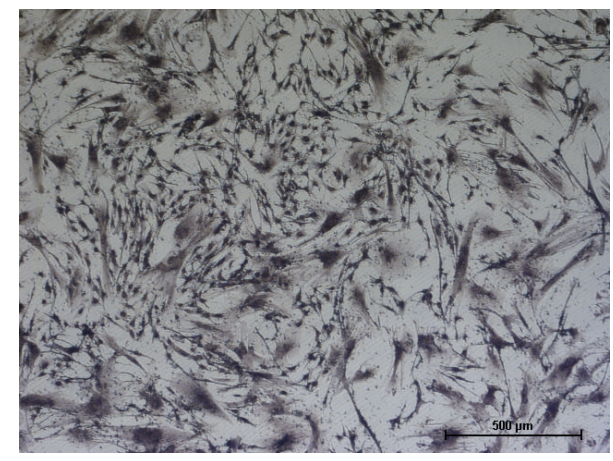

(b)

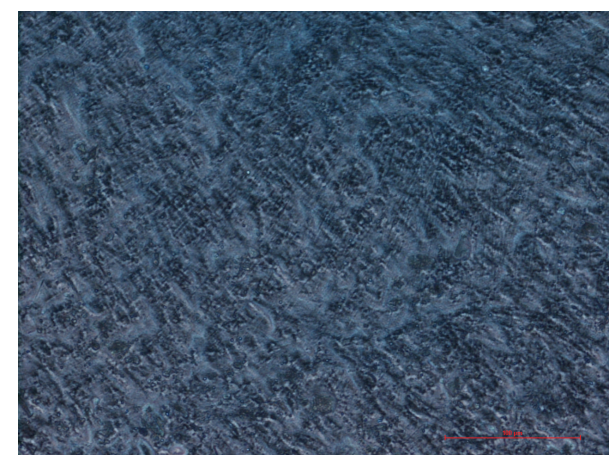

(d)

FIgURE 3: Multipotential differentiation of MB-MSCs. (a) Adipocytic differentiation. Red indicated lipid vacuoles stained by Oil-Red. (b) Osteocytic differentiation. Purple indicated alkaline phosphatases stained by ALP staining. (c, d) Chondrocytic differentiation. Blue indicated extracellular matrix component glycosaminoglycans stained by Alcian Blue.

of MB-MSCs based on their different densities [10]. However, there are a variety of cells in menstrual blood with different densities; thus, the use of density gradient centrifugation can result in loss of MB-MSCs. As such, we used adherence for isolation of MSCs from menstrual blood. It allows for quick adherence and cell growth, and nonadherent hematopoietic cells and dead cells can be removed with the change of culture medium. In addition, there is no significant difference in purity. It has been suggested that a culture system using a small amount of fibroblasts as feeder cells allows the production and survival of embryonic stem cells due to the synergistic effect of the secretion of growth factors [24]. Thus, adherence could be a promising alternative to the density gradient centrifugation for the isolation of MSCs from menstrual blood.

We also investigated the growth of MB-MSCs to characterize their proliferative capacity. It was observed that MBMSCs could be cultured for 10-15 sequential passages (more than 20 months). After freezing and thawing, they still preserved proliferation capacity and clonogenicity. The results of this study showed that MB-MSCs could be subcultured for up to 35 passages, which was in agreement with the findings of Allickson et al. [25], indicating that MB-MSCs were highly proliferative. This may be associated with telomerase activity [26]. Telomerases serve to protect chromosome ends from end-to-end fusion, recombination, and degradation. It has been shown that knock-out of telomerase leads to telomere shortening and chromosomal abnormalities, thus reducing the proliferative capacity of stem cells [27].

We found that MB-MSCs could secrete VEGF, HGF, and IGF-1 that played a key role in tissue repair and regeneration. Rehman et al. [28] showed that adipose tissue-derived MSCs could secrete antiapoptotic factors, such as VEGF, HGF, and IGF-1. Tögel et al. [29] confirmed that these factors improved kidney function through the bypass secretion, rather than differentiating into target cells. Fu et al. [30] showed that MSCs produced a wide array of cytokines, such as VEGF, IGF-1, and HGF, and direct injection of BM-MSCs into ovary enhanced ovarian function in rats with chemotherapy-induced ovarian damage. These effects appear to be mediated by the paracrine mechanisms of cytokines. MSCs are present in bone marrow, peripheral blood, cord blood, fat, brain, thymus, muscle, liver, and lung, but the use of MSCs could be limited due to invasiveness of extraction and proliferative capacity. It is clear that MSCs from menstrual blood offer certain advantages including noninvasive and ethically acceptable availability; thus, they may be a practical solution for stem cell therapy.

In conclusion, we successfully isolated MSCs from menstrual blood using adherence and investigated their biological characteristics, proliferative capacity and secretion levels. MB-MSCs showed a high multilineage potential and clonogenic activity and secreted a large amount of VEGF, HGF, and IGF-1. These factors might play a key role in tissue repair and regeneration. In comparison with MSCs from other sources, 


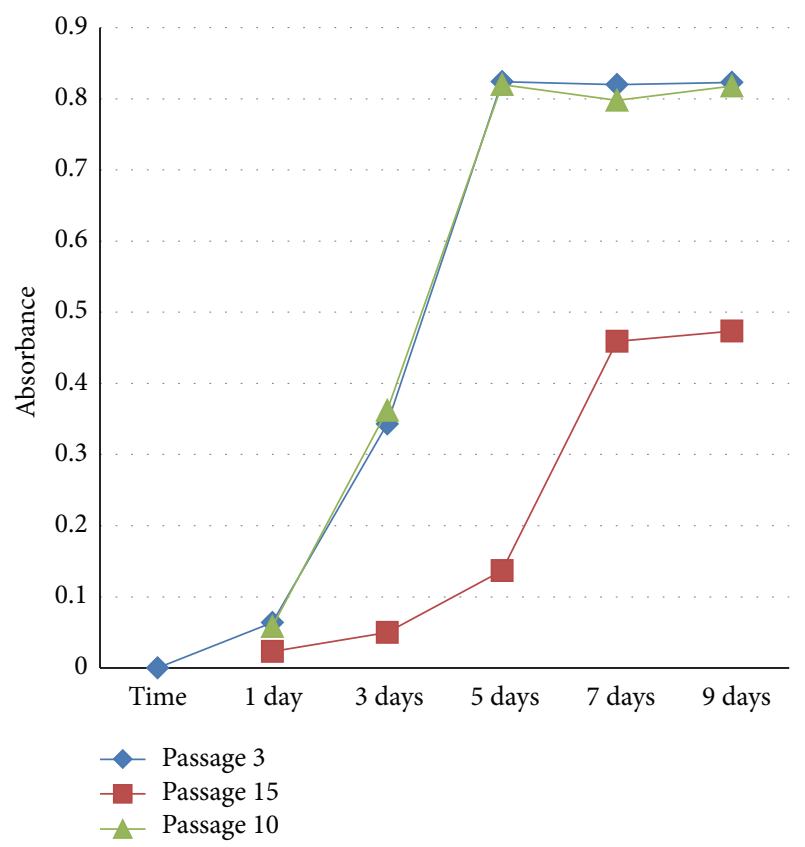

FIGURE 4: Growth curves of subcultured MB-MSCs.

a significant advantage of MB-MSCs is their easy availability, thus avoiding invasive procedures and ethical controversies. Isolation of MSCs from menstrual blood using adherence is very simple and time saving. However, there are still many problems to be solved, such as surface markers with sufficient specificity to identify MB-MSCs and the source of MB-MSCs. We believe that this cell population could become a practical solution for autologous stem cell therapy.

\section{Conflict of Interests}

The authors declare that there is no conflict of interests regarding the publication of this paper.

\section{Authors' Contribution}

Xue Du and Qing Yuan contributed equally to this work.

\section{Acknowledgment}

This work was supported by grants ( 81303108 to Xue Du) from the National Natural Science Foundation of China.

\section{References}

[1] E. F. Wolff, X.-B. Gao, K. V. Yao et al., "Endometrial stem cell transplantation restores dopamine production in a Parkinson's disease model," Journal of Cellular and Molecular Medicine, vol. 15, no. 4, pp. 747-755, 2011.

[2] M. Morigi, B. Imberti, C. Zoja et al., "Mesenchymal stem cells are renotropic, helping to repair the kidney and improve function in acute renal failure," Journal of the American Society of Nephrology, vol. 15, no. 7, pp. 1794-1804, 2004.
[3] C. Roufosse and H. T. Cook, "Stem cells and renal regeneration," Nephron-Experimental Nephrology, vol. 109, no. 2, pp. e39-e45, 2008.

[4] M. T. Abdel Aziz, H. M. Atta, S. Mahfouz et al., “Therapeutic potential of bone marrow-derived mesenchymal stem cells on experimental liver fibrosis," Clinical Biochemistry, vol. 40, no. 12, pp. 893-899, 2007.

[5] M. F. Pittenger, A. M. Mackay, S. C. Beck et al., "Multilineage potential of adult human mesenchymal stem cells," Science, vol. 284, no. 5411, pp. 143-147, 1999.

[6] R. W. S. Chan, K. E. Schwab, and C. E. Gargett, "Clonogenicity of human endometrial epithelial and stromal cells," Biology of Reproduction, vol. 70, no. 6, pp. 1738-1750, 2004.

[7] X. Meng, T. E. Ichim, J. Zhong et al., "Endometrial regenerative cells: a novel stem cell population," Journal of Translational Medicine, vol. 5, article 57, 2007.

[8] V. A. Prianishnikov, "On the concept of stem cell and a model of functional-morphological structure of the endometrium," Contraception, vol. 18, no. 3, pp. 213-223, 1978.

[9] A. N. Patel, E. Park, M. Kuzman, F. Benetti, F. J. Silva, and J. G. Allickson, "Multipotent menstrual blood stromal stem cells: isolation, characterization, and differentiation," Cell Transplantation, vol. 17, no. 3, pp. 303-311, 2008.

[10] R. A. Musina, A. V. Belyavski, O. V. Tarusova, E. V. Solovyova, and G. T. Sukhikh, "Endometrial mesenchymal stem cells isolated from the menstrual blood," Bulletin of Experimental Biology and Medicine, vol. 145, no. 4, pp. 539-543, 2008.

[11] C. V. Borlongan, Y. Kaneko, M. Maki et al., "Menstrual blood cells display stem cell-like phenotypic markers and exert neuroprotection following transplantation in experimental stroke," Stem Cells and Development, vol. 19, no. 4, pp. 439-452, 2010.

[12] Z. Jiang, X. Hu, H. Yu et al., "Human endometrial stem cells confer enhanced myocardial salvage and regeneration by paracrine mechanisms," Journal of Cellular and Molecular Medicine, vol. 17, no. 10, pp. 1247-1260, 2013.

[13] C. E. Gargett, "Uterine stem cells: what is the evidence?" Human Reproduction Update, vol. 13, no. 1, pp. 87-101, 2007.

[14] H. L. Du and H. S. Taylor, "Contribution of bone marrowderived stem cells to endometrium and endometriosis," Stem Cells, vol. 25, no. 8, pp. 2082-2086, 2007.

[15] H. S. Taylor, "Endometrial cells derived from donor stem cells in bone marrow transplant recipients," Journal of the American Medical Association, vol. 292, no. 1, pp. 81-85, 2004.

[16] J. J. Minguell, A. Erices, and P. Conget, "Mesenchymal stem cells," Experimental Biology and Medicine, vol. 226, no. 6, pp. 507-520, 2001

[17] S. A. Kuznetsov, M. H. Mankani, S. Gronthos, K. Satomura, P. Bianco, and P. G. Robey, "Circulating skeletal stem cells," Journal of Cell Biology, vol. 153, no. 5, pp. 1133-1140, 2001.

[18] N. J. Zvaifler, L. Marinova-Mutafchieva, G. Adams et al., "Mesenchymal precursor cells in the blood of normal individuals," Arthritis Research, vol. 2, no. 6, pp. 477-488, 2000.

[19] R. Hass, C. Kasper, S. Böhm, and R. Jacobs, "Different populations and sources of human mesenchymal stem cells (MSC): a comparison of adult and neonatal tissue-derived MSC," Cell Communication and Signaling, vol. 9, article 12, 2011.

[20] M. Körbling and Z. Estrov, "Adult stem cells for tissue repaira new therapeutic concept?" The New England Journal of Medicine, vol. 349, no. 6, pp. 570-582, 2003.

[21] M. Dominici, K. Le Blanc, I. Mueller et al., "Minimal criteria for defining multipotent mesenchymal stromal cells. The 
International Society for Cellular Therapy position statement," Cytotherapy, vol. 8, no. 4, pp. 315-317, 2006.

[22] N. Hida, N. Nishiyama, S. Miyoshi et al., "Novel cardiac precursor-like cells from human menstrual blood-derived mesenchymal cells," Stem Cells, vol. 26, no. 7, pp. 1695-1704, 2008.

[23] M. Toyoda, C. Cui, and A. Umezawa, "Myogenic transdifferentiation of menstrual blood-derived cells," Acta Myologica, vol. 26, no. 3, pp. 176-178, 2007.

[24] O. Hovatta, M. Mikkola, K. Gertow et al., "A culture system using human foreskin fibroblasts as feeder cells allows production of human embryonic stem cells," Human Reproduction, vol. 18, no. 7, pp. 1404-1409, 2003.

[25] J. G. Allickson, A. Sanchez, N. Yefimenko, C. V. Borlongan, and P. R. Sanberg, "Recent studies assessing the proliferative capability of a novel adult stem cell identified in menstrual blood," Open Stem Cell Journal, vol. 3, pp. 4-10, 2011.

[26] R. C. Allsopp, G. B. Morin, R. DePinho, C. B. Harley, and I. L. Weissman, "Telomerase is required to slow telomere shortening and extend replicative lifespan of HSCs during serial transplantation," Blood, vol. 102, no. 2, pp. 517-520, 2003.

[27] J. Nichols, B. Zevnik, K. Anastassiadis et al., "Formation of pluripotent stem cells in the mammalian embryo depends on the POU transcription factor Oct4," Cell, vol. 95, no. 3, pp. 379391, 1998.

[28] J. Rehman, D. Traktuev, J. Li et al., "Secretion of angiogenic and antiapoptotic factors by human adipose stromal cells," Circulation, vol. 109, no. 10, pp. 1292-1298, 2004.

[29] F. Tögel, K. Weiss, Y. Yang, Z. Hu, P. Zhang, and C. Westenfelder, "Vasculotropic, paracrine actions of infused mesenchymal stem cells are important to the recovery from acute kidney injury," American Journal of Physiology-Renal Physiology, vol. 292, no. 5, pp. F1626-F1635, 2007.

[30] X. Fu, Y. He, C. Xie, and W. Liu, "Bone marrow mesenchymal stem cell transplantation improves ovarian function and structure in rats with chemotherapy-induced ovarian damage," Cytotherapy, vol. 10, no. 4, pp. 353-363, 2008. 

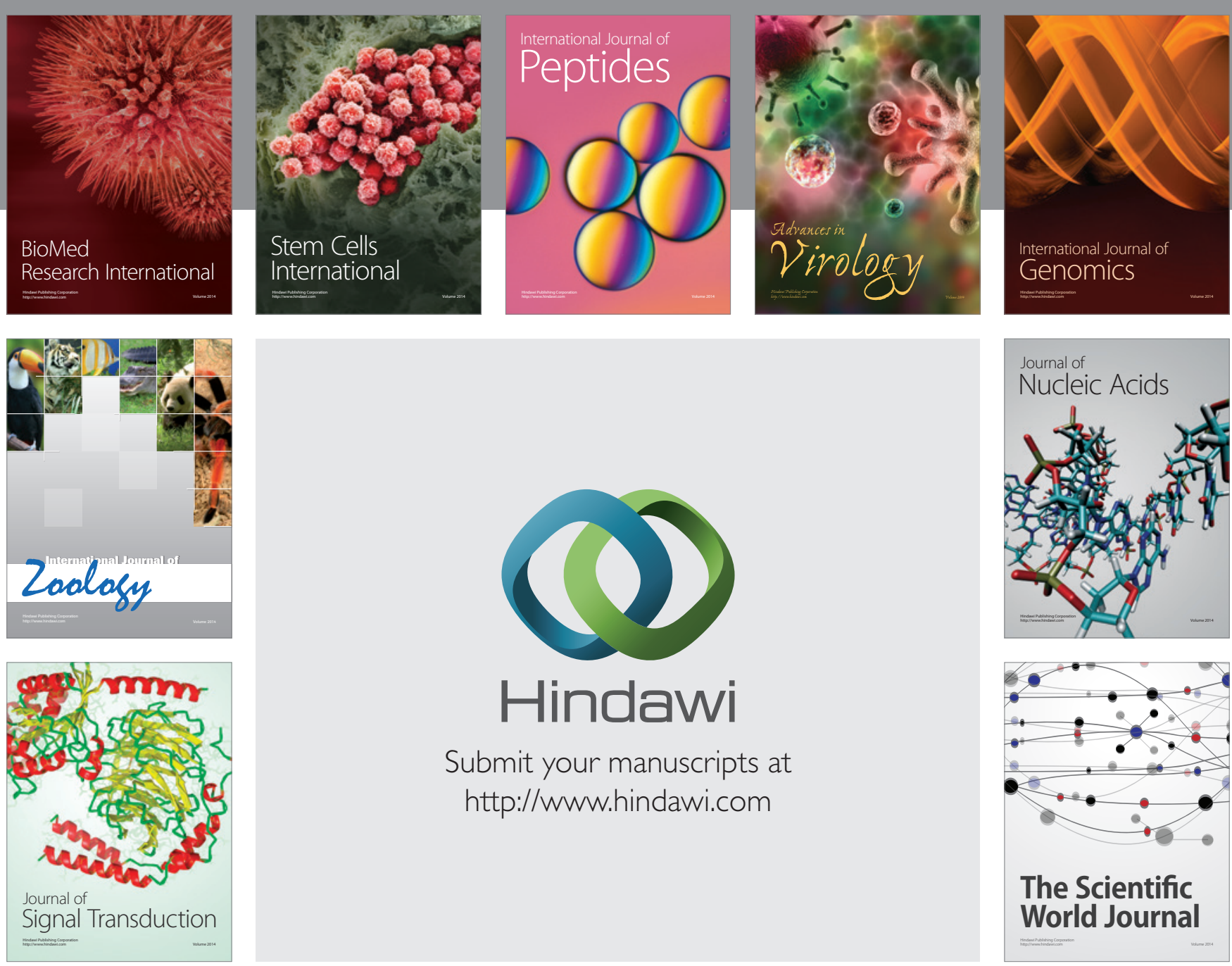

Submit your manuscripts at

http://www.hindawi.com
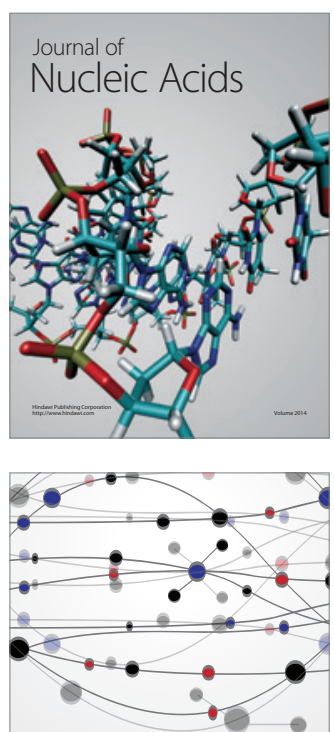

The Scientific World Journal
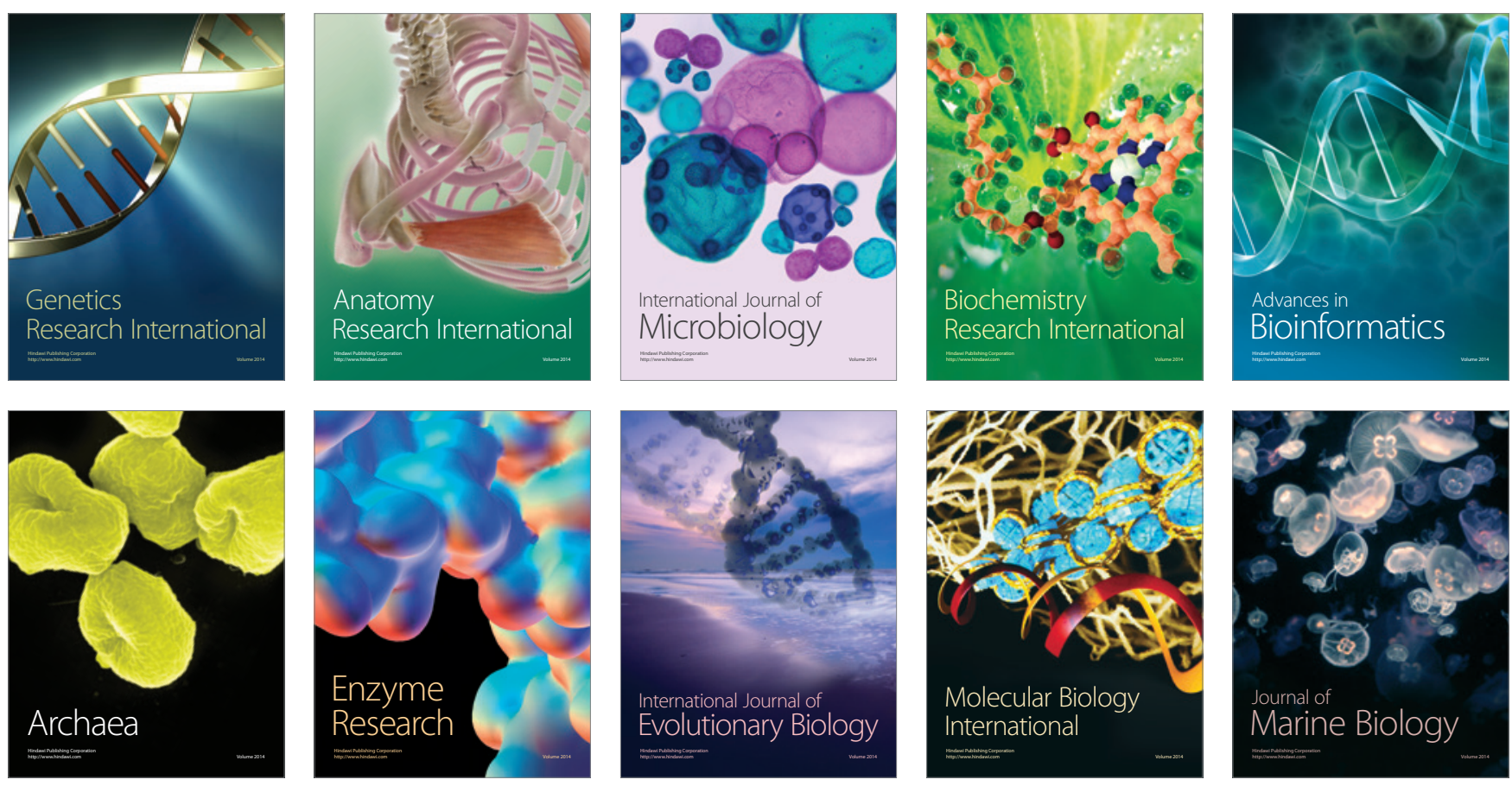